\title{
LKB1 maintains the balance
}

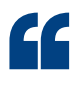

LKB1 balances proliferation and quiescence in HSCs

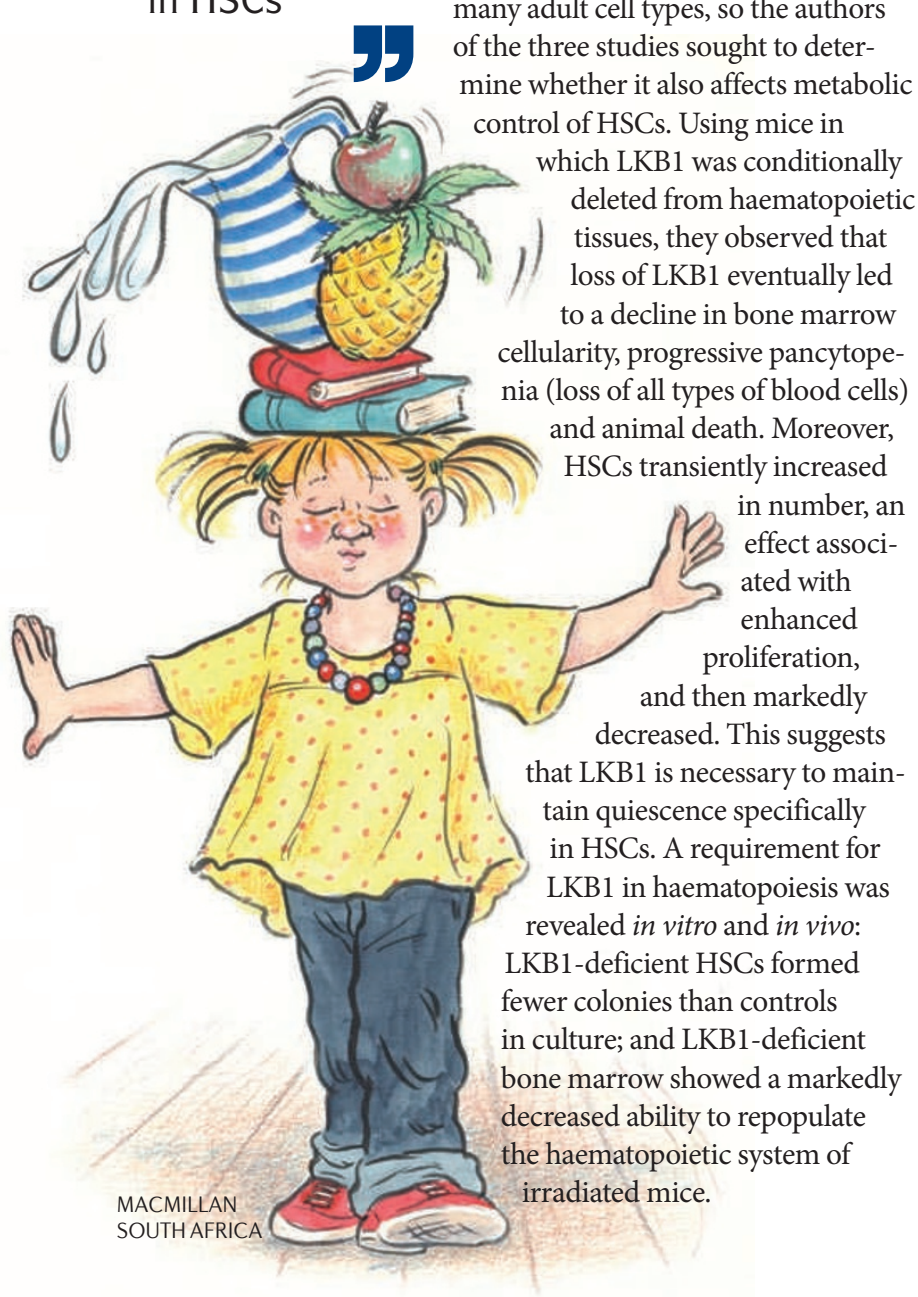

The Ser/Thr kinase LKB1 (also known as STK11) is essential for the maintenance of haematopoietic stem cell (HSC) homeostasis, according to three studies published in Nature.

$\mathrm{LKB} 1$ regulates the metabolism of many adult cell types, so the authors of the three studies sought to determine whether it also affects metabolic which LKB1 was conditionally deleted from haematopoietic tissues, they observed that loss of LKB1 eventually led cellularity, progressive pancytopean
The decrease in HSC numbers . Indeed, Gan et al. observed that LKB1-deficient HSCs have reduced levels of PPAR $\gamma$ co-activator 1 (PGC1), a transcriptional co-activator with known roles in metabolism and mitochondrial biogenesis. Consistent with this, all three studies show that loss of LKB1 leads to reduced ATP levels and decreased mitochondrial potential.

LKB1 is known to limit cell growth in the absence of nutrients by phosphorylating AMP-activated protein kinase (AMPK), thereby inhibiting mammalian target of rapamycin complex 1 (mTORC1) signalling. However, the three studies find that the downstream effects of LKB1 are mTORC1 independent, as treatment with the mTORC1 inhibitor rapamycin did not did not rescue the effects of LKB1 deficiency, including HSC depletion. Furthermore, Gurumurthy et al. and Gan et al. observed that treatment with an AMPK activator did not restore the depleted cells in the bone marrow and thymus, and Nakada et al. found that AMPK-deficient HSCs were neither rapidly depleted nor unable to reconstitute irradiated mice, in contrast to LKB1-deficient HSCs. Thus, most of the effects of LKB1 on HSC homeostasis are independent of AMPK and mTORC1.

So, it seems that LKB1 balances proliferation and quiescence in HSCs by regulating cell survival, cell cycle progression and mitochondrial function. Candidate downstream effectors of LKB1 signalling include AMPK-related kinases, and further studies are needed to determine whether they are involved in this LKB1-mediated function.

Rachel David

ORIGINAL RESEARCH PAPERS Nakada, D., Saunders, T. L. \& Morrison, S. J. Lkb1 regulates cell cycle and energy metabolism in haematopoietic stem cells. Nature 468, 653-658 (2010)| Gurumurthy, S. et al. The Lkb1 metabolic sensor maintains haematopoietic stem cell survival. Nature 468, 659-663 (2010) | Gan, B. et al. Lkb1 regulates quiescence and metabolic homeostasis of haematopoietic stem cells. Nature 468, 701-704 (2010) 